\title{
Very Colorful Theorems
}

\author{
Jorge L. Arocha • Imre Bárány • Javier Bracho • \\ Ruy Fabila $\cdot$ Luis Montejano
}

Received: 20 March 2008 / Revised: 25 March 2009 / Accepted: 8 April 2009 /

Published online: 8 May 2009

(C) Springer Science+Business Media, LLC 2009

\begin{abstract}
We prove several colorful generalizations of classical theorems in discrete geometry. Moreover, the colorful generalization of Kirchberger's theorem gives a generalization of the theorem of Tverberg on non-separated partitions.
\end{abstract}

Keywords Convex sets · Colorful versions · Theorems of Helly · Carathéodory · Kirchberger · Tverberg and Hadwiger

J.L. Arocha $(\bowtie) \cdot$ J. Bracho $\cdot$ R. Fabila $\cdot$ L. Montejano Instituto de Matemáticas, Universidad Nacional Autónoma de México, Ciudad Universitaria, México DF 04510, Mexico

e-mail: arocha@math.unam.mx

J. Bracho

e-mail: roli@math.unam.mx

R. Fabila

e-mail: ruyfabila@gmail.com

L. Montejano

e-mail: luis@math.unam.mx

I. Bárány

Renyi Institute of Mathematics, Hungarian Academy of Sciences, P.O. Box 127, 1364 Budapest, Hungary

e-mail: barany@ math-inst.hu

I. Bárány

Department of Mathematics, University College London, Gower Street, London WC1E 6BT,

England, UK

L. Montejano

Facultad de Matemáticas, Universidad Autónoma de Guerrero, Acapulco, Guerrero, Mexico 


\section{Introduction}

A prominent role in combinatorial geometry is played by Helly's theorem, which states that a finite family of convex sets in $\mathbb{R}^{d}$ has a non-empty intersection if and only if every subfamily of size $d+1$ has a non-empty intersection. Results of the type "if every subfamily of size $k$ of a family $\mathcal{A}$ has some property $\mathcal{P}$, then the whole family also has the property $\mathcal{P}$ " are called Helly-type theorems and have been the object of active research.

Associated with every Helly-type theorem we have a colorful version. Suppose in addition that every object of $\mathcal{A}$ is painted with at least one of $k$ colors. Assume that every subset of $\mathcal{A}$ of size $k$, that uses the $k$ different colors, has property $\mathcal{P}$. It is too much to expect, then, that the whole family $\mathcal{A}$ also has property $\mathcal{P}$. What usually happens, but not always, ${ }^{1}$ is that there is a color $i$ such that the subfamily of all elements of color $i$ has property $\mathcal{P}$.

In this sense, the first colorful theorem was discovered by Lovász, and it is the colorful version of Helly's theorem. Independently, searching for a mathematical game, Bárány found the Colorful Carathéodory Theorem [2]. To be more precise: if a finite set $A \subset \mathbb{R}^{d}$ is colored with $d+1$ colors and $x \in \mathbb{R}^{d}$ is separated from every colorful subset of size $d+1$, then there is a color such that $x$ is separated from the set of all points of this color. Here two sets of points $A, B$ are separated if $\operatorname{conv} A \cap \operatorname{conv} B=\varnothing$. In the same spirit, there are colorful versions of several classical transversal theorems; see $[1,5]$.

In this paper we will prove several generalizations of colorful theorems which we will call "very colorful" to distinguish them from the older ones. In Sect. 2 we will use topology to prove that, in fact, for the colorful version of Carathéodory's theorem, we have as a conclusion that there exist two colors such that $x$ is separated from the set of all points of some of those colors.

There is a very interesting and less known Helly-type theorem, namely Kirchberger's theorem. Suppose that we have two kinds of points $A, B \subset \mathbb{R}^{d}$ say square and round points. Kirchberger's theorem asserts that if for every subset of $A \cup B$ of size $d+2$, the square points and the round points are separated, then the same is true for the whole set $A \cup B$.

In Sect. 3 we give several generalizations of Kirchberger's theorem. First of all, the $r$-partite version of this theorem is true, where the notion of separability is the natural one and the "magic" number used for this theorem is the Tverberg number $(r-1)(d+1)+1$. Second, this result has a colorful version. Furthermore, this colorful version has, as a special case, Tverberg's theorem, which is the $r$-partite version of Radon's theorem and one of the most beautiful results in combinatorial geometry. Its proof has two ingredients. The first is the Colorful Carathéodory Theorem [2] (only one color in the conclusion). The second, which is based on an idea of Sarkaria [7], is a necessary and sufficient condition for $\bigcap_{i=1}^{r}$ conv $A_{i}=\varnothing\left(\right.$ where $A_{i} \subset \mathbb{R}^{d}$ ) in terms of a well-defined tensor product. If we use more of the strength of the Very Colorful

\footnotetext{
${ }^{1}$ The theorem "if every four points in $A \subset \mathbb{R}^{2}$ are in convex position then all points of $A$ are in convex position" has no colorful version.
} 
Carathéodory Theorem (two colors in the conclusion), then we end up with an interesting non-trivial generalization of the theorems of Tverberg and Radon (Sect. 4).

In Sect. 5 we use the Very Colorful Carathéodory Theorem to show that in the conclusion of the spherical (cone) version of the Colorful Helly Theorem the existence of two colors can be ensured. This is not the case for Lovász's Colorful Helly Theorem, but we will generalize it in another direction: if one asks that every subfamily with a large number of colors (not only the colorful ones) is intersecting, then the number of intersecting colors increases.

Again, take a Helly-type theorem (if every subfamily of size $k$ of a family $\mathcal{A}$ has some property $\mathcal{P}$, then the whole family also has property $\mathcal{P}$ ) and suppose now that every object of the family $\mathcal{A}$ is painted with at least one of $m \geq k$ colors. Suppose also that every colorful subfamily of size $k$ of $\mathcal{A}$ has property $\mathcal{P}$. Then what usually happens is that there is a number of colors with the property that the set of all elements of these colors also satisfies property $\mathcal{P}$. Every colorful theorem can be generalized in this way. Usually, this generalization does not follow directly from the colorful version but can be proved with an easy modification of the original proof. This is the case for all colorful theorems in Sects. 2-5.

However, we found that the modifications needed to prove such a generalization of the Colorful Hadwiger Theorem [1] are not so easy. So, we dedicate the last section of this paper to explain how to modify the known proof of the Colorful Hadwiger Theorem.

Finally, a few words on terminology. All the subspaces, hyperplanes, semispaces, etc. are always through the origin. If not, then we will use the adjective "affine". All colorings are surjective. When a set is colored, it is allowed for an element to receive more than one color. Alternatively, one can think that colorings are functions, but repetitions of elements are allowed. Let $B$ be a subset of a colored set. We say that $B$ is colorful if the coloring restricted to it is injective.

\section{Carathéodory's Theorem}

Theorem 1 (Very Colorful Carathéodory Theorem) Let A be a finite set of points in $\mathbb{R}^{d}$ colored with $d+1$ different colors. If every colorful subset $A^{\prime}$ with $\left|A^{\prime}\right|=d+1$ is separated from the origin, then there exist two colors such that the subset of all points of these colors is separated from the origin.

Proof Let $B \subseteq A$ be the colorful subset with $|B| \leq d$ such that conv $B$ is closest to the origin. Let $\alpha$ be the closest point to the origin in $\operatorname{conv} B$. Denote by $\Sigma$ the solid open ball centered at the origin through $\alpha$. Let $\Gamma$ be the affine hyperplane through $\alpha$ tangent to $\Sigma$ and denote by $\Gamma^{0}$ the open affine semispace bounded by $\Gamma$ which contains the origin.

Let $x \in A$ be such that $B^{\prime}=B \cup x$ is colorful. If $x \in \Gamma^{0}$ then the segment $\overline{\alpha x}$ meets $\Sigma$ and therefore conv $B^{\prime}$ is closer to the origin than conv $B$. This implies that there exists $B^{\prime \prime} \subseteq B$ with $\left|B^{\prime \prime}\right| \leq d$ such that conv $B^{\prime \prime}$ is closer to the origin than conv $B$, contradicting the minimality of $B$. So, $\Gamma^{0}$ does not contain points of colors not present in $B$. The number of such colors is $d+1-|B|$. If $|B|<d$, this proves the theorem. If $|B|=d$, then there is exactly one color missing. 
Fig. 1 The $L_{1}$ and $L_{2}$ half-lines

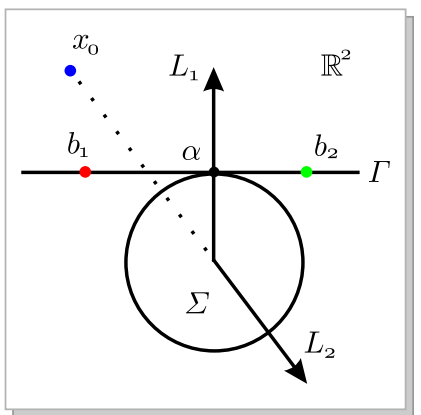

Suppose $|B|=d$ and denote $B=\left\{b_{1}, \ldots, b_{d}\right\}$. If for some color of $B$ there is no point in $A \cap \Gamma^{0}$ of this color, then we are done. So, we can suppose that there is $C=\left\{c_{1}, \ldots, c_{d}\right\} \subseteq A \cap \Gamma^{0}$ such that for every $i$ the point $c_{i}$ has the same color as $b_{i}$.

Let $L_{1}$ be the half-line starting at the origin and containing $\alpha$. Since there are $d+1$ colors, there is a point, $x_{0}$ say, of a color not present in $B$. Let $L_{2}$ be the half-line starting at the origin and containing $-x_{0}$. The situation is shown in Fig. 1.

Let $L=L_{1} \cup L_{2}$. Clearly, the homotopy group $\Pi_{d-2}\left(\mathbb{R}^{d} \backslash L\right)$ is non-zero and the essential $(d-2)$-cycle can be represented by the boundary of the $(d-1)$-simplex conv $B$.

Denote by $E=\left\{e_{1}, \ldots, e_{d}\right\}$ the standard orthonormal basis of $\mathbb{R}^{d}$. Let $\Omega^{d-1}$ be the boundary of the $d$-dimensional cross-polytope which is $\operatorname{conv}\{E \cup-E\}$. Every facet of $\Omega^{d-1}$ is a simplex. A subset of $d$ vertices of $\Omega^{d-1}$ spans a facet if and only if it does not contain antipodal points. For any $i$ we paint $e_{i}$ and $-e_{i}$ with the color of $b_{i}$. Therefore, every facet of $\Omega^{d-1}$ is a colorful $(d-1)$-dimensional simplex. Finally, let $U$ be the interior of the facet conv $E$. So, $\Omega^{d-1} \backslash U$ is a $(d-1)$-dimensional simplicial complex piecewise linear homeomorphic to a $(d-1)$-simplex.

Let $f: \Omega^{d-1} \backslash U \rightarrow \mathbb{R}^{d}$ be the piecewise linear map defined on the vertices of $\Omega^{d-1} \backslash U$ by sending $e_{i}$ to $b_{i} \in B$, sending $-e_{i}$ to $c_{i} \in C$ and then extending linearly. Note that $f$ preserves colors. Since $f$ restricted to the boundary of $\Omega^{d-1} \backslash U$ is by definition the essential $(d-2)$-cycle of $\Pi_{d-2}\left(\mathbb{R}^{d} \backslash L\right)$, the image of $f$ cannot avoid $L$. Therefore, there exists a facet of $\Omega^{d-1} \backslash U$ whose image by $f$ intersects $L$. Let $\sigma=\left\{z_{1}, \ldots, z_{d}\right\} \neq E$ be a set of vertices of $\Omega^{d-1}$ such that $L \cap \operatorname{conv} f(\sigma) \neq \varnothing$.

If $L_{2} \cap$ conv $f(\sigma) \neq \varnothing$, then $f(\sigma) \cup x_{0}$ is a colorful subset of $d+1$ points which is not separated from the origin. This contradicts the hypothesis. If $L_{1} \cap \operatorname{conv} f(\sigma) \neq \varnothing$, then there exists a point $\beta \in L_{1} \cap \operatorname{conv} f(\sigma)$ which is closer to the origin than $\alpha$. This contradicts the minimality of $B$.

Observe that this theorem is sharp in the sense that one can get only two colors and no more. This can be seen from the following example. Take $d+2$ points $\left\{x_{0}, \ldots, x_{d}, y\right\} \subseteq \mathbb{R}^{d}$ such that $\left\{x_{0}, \ldots, x_{d}, y, 0\right\}$ is in general position and $0 \in \operatorname{conv}\left\{x_{0}, \ldots, x_{d}\right\}$. Paint each of the $x_{i}$ with the first $d-1$ colors and paint $y$ with the remaining two colors. The conditions of the theorem are fulfilled and only the colors of $y$ are separated from the origin. 
Fig. 2 The equivalence between Kirchberger's and Carathéodory's theorems

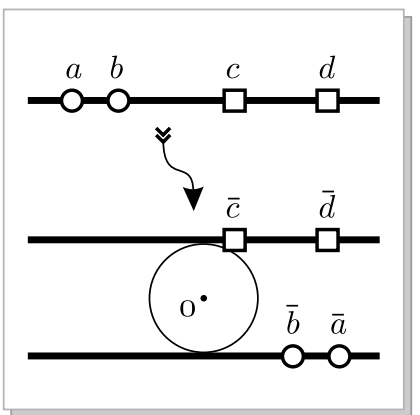

\section{Kirchberger's Theorem}

Let $A$ and $B$ be two finite sets of points in $\mathbb{R}^{d}$ such that $|A|+|B| \geq d+2$. Kirchberger's theorem asserts that conv $A \cap \operatorname{conv} B \neq \varnothing$ if and only if there exist $A^{\prime} \subseteq A$ and $B^{\prime} \subseteq B$ with $\left|A^{\prime}\right|+\left|B^{\prime}\right|=d+2$ such that conv $A^{\prime} \cap$ conv $B^{\prime} \neq \varnothing$. Usually, one asks the sets $A$ and $B$ to be disjoint because if not, then the statement becomes obvious. However, in the colorful version, which we will state below, the theorem makes sense even when $A=B$. Therefore, we will not insist on such a limitation to Kirchberger's theorem.

Kirchberger's theorem follows from Carathéodory's theorem using the following construction. Denote $\tilde{x}=\left(\begin{array}{l}x \\ 1\end{array}\right) \in \mathbb{R}^{d+1}$. If $x \in A$, then we code this point by the vector $\bar{x}=\tilde{x}$ and if $x \in B$, then we code this point by the vector $\bar{x}=-\widetilde{x}$. It turns out that conv $A \cap$ conv $B \neq \varnothing$ if and only if $\{\bar{x} \mid x \in A \cup B\}$ is separated from the origin. This construction is shown for a particular case in Fig. 2.

We will prove this fact in a more general setting when there are $r \geq 2$ sets of points. Let $\mathcal{A}=\left\{A_{1}, \ldots, A_{r}\right\}$ be a family of finite sets of vectors in $\mathbb{R}^{d}$. We say that $\mathcal{A}$ is separated if $\bigcap_{1}^{r} \operatorname{conv} A_{i}=\varnothing$.

Let $v_{1}, \ldots, v_{r}$ be vectors in $\mathbb{R}^{r-1}$ with a unique linear dependence $v_{i}+\cdots+$ $v_{r}=0$. For $x \in A_{i}$ denote $\tilde{x}=\left(\begin{array}{l}x \\ 1\end{array}\right) \in \mathbb{R}^{d+1}$ and $\bar{x}=\tilde{x} \otimes v_{i} \in \mathbb{R}^{(r-1)(d+1)}$. Denote also $\bar{X}=\bigcup_{i=1}^{r}\left\{\bar{x} \mid x \in A_{i}\right\}$.

Lemma 2 (Sarkaria [7]) $\mathcal{A}$ is separated if and only if $0 \notin \operatorname{conv} \bar{X}$.

Proof We show that $\mathcal{A}$ is not separated if and only if $0 \in \operatorname{conv} \bar{X}$. We start by assuming that $0 \in \operatorname{conv} \bar{X}$, i.e., there exist positive real numbers $\alpha(x)$ with $\sum \alpha(x)=1$ such that

$$
0=\sum_{i=1}^{r} \sum_{x \in A_{i}} \alpha(x) \bar{x}=\sum_{i=1}^{r} \sum_{x \in A_{i}} \alpha(x) \tilde{x} \otimes v_{i}=\sum_{i=1}^{r}\left(\sum_{x \in A_{i}} \alpha(x) \tilde{x}\right) \otimes v_{i} .
$$

There exists a unique vector $u \in \mathbb{R}^{r-1}$ which is the solution of the system of linear equations $u v_{1}=1, u v_{2}=-1$ and $u v_{i}=0$ for $i>2$. Multiplying from the right by $u$ gives

$$
\sum_{x \in A_{1}} \alpha(x) \tilde{x}=\sum_{x \in A_{2}} \alpha(x) \tilde{x},
$$


and similarly we get

$$
\sum_{x \in A_{1}} \alpha(x) \tilde{x}=\sum_{x \in A_{2}} \alpha(x) \tilde{x}=\cdots=\sum_{x \in A_{r}} \alpha(x) \tilde{x},
$$

which is equivalent to

$$
\begin{gathered}
\sum_{x \in A_{1}} \alpha(x) x=\sum_{x \in A_{2}} \alpha(x) x=\cdots=\sum_{x \in A_{r}} \alpha(x) x, \\
\sum_{x \in A_{1}} \alpha(x)=\sum_{x \in A_{2}} \alpha(x)=\cdots=\sum_{x \in A_{r}} \alpha(x)=\frac{1}{r},
\end{gathered}
$$

and therefore $r \sum_{x \in A_{1}} \alpha(x) x$ is a common point of all conv $A_{i}$.

Conversely, if $r \sum_{x \in A_{1}} \alpha(x) x$ is a common point of all conv $A_{i}$, then for every $x$ in every $A_{i}$ there exists a positive scalar $\alpha(x)$ such that the equalities (2) hold. Tensorially multiplying the equality $v_{i}+\cdots+v_{r}=0$ from the left by $\sum_{x \in A_{1}} \alpha(x) \tilde{x}$, we obtain the equalities (1) and therefore $0 \in \operatorname{conv} \bar{X}$.

For two families of sets $\mathcal{A}=\left\{A_{1}, \ldots, A_{r}\right\}$ and $\mathcal{B}=\left\{B_{1}, \ldots, B_{r}\right\}$ we will say that $\mathcal{A}$ is smaller than $\mathcal{B}$ if for all $i \in\{1, \ldots, r\}$ the inclusion $A_{i} \subseteq B_{i}$ holds. If $\mathcal{A}$ is smaller than $\mathcal{B}$ then we will write $\mathcal{A} \preceq \mathcal{B}$.

For a family of sets $\mathcal{A}=\left\{A_{1}, \ldots, A_{r}\right\}$ we will denote by $\sqcup \mathcal{A}$ the disjoint union of the $A_{i}$, i.e., $\sqcup \mathcal{A}$ is $\bigcup_{1}^{r} A_{i}$ but points may be repeated. If $\sqcup \mathcal{A}$ is colored, then we will say that $\mathcal{A}^{\prime} \preceq \mathcal{A}$ is colorful if the coloring restricted to $\sqcup \mathcal{A}^{\prime}$ is injective. Moreover, for any set of colors $\nabla$ let $\mathcal{A}[\nabla]$ denote the family $\left\{A_{1}^{\prime}, \ldots, A_{r}^{\prime}\right\}$, where $A_{i}^{\prime}$ is the set of all elements of $A_{i}$ whose color is in $\nabla$.

Theorem 3 (Very Colorful Kirchberger Theorem) Let $\mathcal{A}=\left\{A_{1}, \ldots, A_{r}\right\}$ be a family of finite sets of points in $\mathbb{R}^{d}$. Denote $n=(r-1)(d+1)$. Suppose that $\sqcup \mathcal{A}$ is colored with $n+1$ different colors. If every colorful smaller family $\mathcal{A}^{\prime} \preceq \mathcal{A}$ with $\left|\sqcup \mathcal{A}^{\prime}\right|=n+1$ is separated, then there are two colors $\alpha$ and $\beta$ such that $\mathcal{A}[\alpha, \beta]$ is separated.

Proof For $x \in \sqcup \mathcal{A}$ denote $\bar{x}$ as Sarkaria's lemma. The color of $\bar{x}$ is the same as that of $x$. By Sarkaria's lemma, every family $\mathcal{A}^{\prime} \preceq \mathcal{A}$ is separated if and only if $\overline{\sqcup \mathcal{A}^{\prime}}=$ $\left\{\bar{x} \mid x \in \sqcup \mathcal{A}^{\prime}\right\}$ is separated from the origin. Applying the Very Colorful Carathéodory

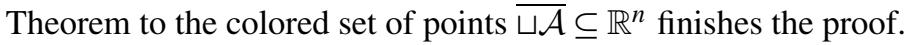

Observe that, if one has to show the existence of just one color, then it is enough to apply the Colorful Carathéodory Theorem [2] instead of Theorem 1. This will be the case in applications given by Corollary 4 and Theorem 5 .

Corollary 4 (Multipartite Kirchberger) Let $\mathcal{A}=\left\{A_{1}, \ldots, A_{r}\right\}$ be a partition of a finite set $X$ of points in $\mathbb{R}^{d}$. The partition $\mathcal{A}$ is not separated if and only if there is a set $X^{\prime} \subseteq X$ with $\left|X^{\prime}\right|=(r-1)(d+1)+1$ such that $\left\{A_{1} \cap X^{\prime}, \ldots, A_{r} \cap X^{\prime}\right\}$ is not separated. 
Proof One implication follows from Theorem 3 taking $(r-1)(d+1)+1$ colors and painting each point in $X$ with all colors. The other implication is obvious.

We mention that the Multipartite Kirchberger theorem was proved first by Attila Pór [6] in his university thesis.

\section{Tverberg's Theorem}

The Very Colorful Kirchberger Theorem is a powerful theorem, and some of its particular cases deserve extra discussion. Let $\mathcal{A}=\left\{A_{1}, \ldots, A_{r}\right\}$, where $A_{1}=A_{2}=\cdots=$ $A_{r}=A$ and $A=\left\{a_{1}, \ldots, a_{n+1}\right\}$ is some set of $n+1=(r-1)(d+1)+1$ points in $\mathbb{R}^{d}$. We paint the point $a_{i}$ with color $i$ in every set $A_{j}$. It is clear that the family $\mathcal{A}[i]$ is non-separated for every color $i$. So by the Very Colorful Kirchberger Theorem (one color in the conclusion) there must be a colorful family $\mathcal{B}$ and $\mathcal{B}=\left\{B_{1}, \ldots, B_{r}\right\} \preceq \mathcal{A}$ with $|\sqcup \mathcal{B}|=n+1$ which is not separated. By its properties, $\mathcal{B}$ is a partition of $A$ and no $B_{i}$ can be empty. Rephrasing this case, we obtain Tverberg's theorem.

Theorem 5 (Tverberg) Every set of $(r-1)(d+1)+1$ points in $\mathbb{R}^{d}$ has a nonseparated partition into $r$ parts.

The preceding proof of Tverberg's theorem uses little of the strength of the Very Colorful Kirchberger Theorem in particular; the latter concludes that there exists a set $\nabla$ of not only one but two colors such that $\mathcal{A}[\nabla]$ is separated. This is not used in Tverberg's theorem. To remedy this situation we consider a more general particular case. Let $\Delta^{n}$ denote the $n$-dimensional simplex.

Theorem 6 (Generalized Tverberg) Denote $n=(r-1)(d+1)$. Suppose that $f_{1}, \ldots, f_{r}: \Delta^{n} \rightarrow \mathbb{R}^{d}$ are linear maps such that for every edge $\sigma^{1} \subseteq \Delta^{n}$ the equality $f_{1}\left(\sigma^{1}\right) \cap \cdots \cap f_{r}\left(\sigma^{1}\right) \neq \varnothing$ holds. Then there exist disjoint faces $\phi_{1}, \ldots, \phi_{r}$ of $\Delta^{n}$ such that $f_{1}\left(\phi_{1}\right) \cap \cdots \cap f_{r}\left(\phi_{r}\right) \neq \varnothing$ and $\sum \operatorname{dim} \phi_{i}=n+1-r$.

Proof Let $\mathcal{A}=\left\{A_{1}, \ldots, A_{r}\right\}$, where $A_{i}=\left\{a_{i, 1}, \ldots, a_{i, n+1}\right\} \subseteq \mathbb{R}^{d}$. For every $i \in$ $\{1, \ldots, r\}$ we color $a_{i j}$ with the color $j$. So the set $A_{i}$ can be interpreted as a function $f_{i}:\{1, \ldots, n+1\} \rightarrow \mathbb{R}^{d}$. The vertices of $\Delta^{n}$ can be identified with the $n+1$ colors. Then $\Delta^{n}$ is the simplex of colors. Extend linearly $f_{i}: \Delta^{n} \rightarrow \mathbb{R}^{d}$. A separated family $\mathcal{A}^{\prime} \preceq \mathcal{A}$ is just a choice of $r$ faces $\phi_{1}, \ldots, \phi_{r}$ of the simplex of colors such that $f_{1}\left(\phi_{1}\right) \cap \cdots \cap f_{r}\left(\phi_{r}\right)=\varnothing$. If $\mathcal{A}^{\prime}$ is colorful, then the faces $\phi_{1}, \ldots, \phi_{r}$ are disjoint. If $\left|\sqcup \mathcal{A}^{\prime}\right|=n+1$, then $\sum \operatorname{dim} \phi_{i}=n+1-r$. Finally, the statement of the Very Colorful Kirchberger Theorem translates into the existence of an edge (spanned by two colors) $\sigma^{1} \subseteq \Delta^{n}$ such that $f_{1}\left(\sigma^{1}\right) \cap \cdots \cap f_{r}\left(\sigma^{1}\right)=\varnothing$. Now if the statement of the Very Colorful Kirchberger Theorem fails for every edge $\sigma^{1} \subset \Delta^{n}$, then its condition has to fail for some colorful and separated family $\mathcal{A}^{\prime} \preceq \mathcal{A}$, finishing the proof.

At first glance, the hypothesis of Theorem 6 seems to be too strong. For instance, when $d>2, f_{1}\left(\sigma^{1}\right)$ and $f_{2}\left(\sigma^{1}\right)$ are disjoint for generic affine maps $f_{1}, f_{2}$. Yet the 
Fig. 3 Two projections of the tetrahedron into the plane

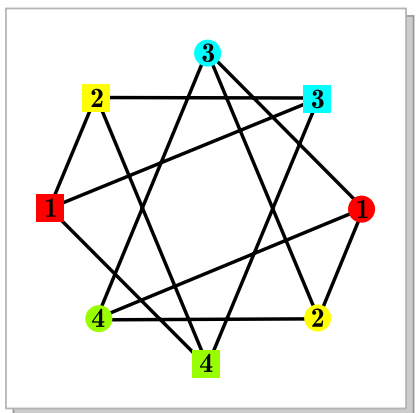

conditions are not excessively strong, at least in the sense of the following example. When $r=2$ and $d=2$, Theorem 6 is a generalization of Radon's theorem in the plane: if $\square$, $\mathrm{O}: \Delta^{3} \rightarrow \mathbb{R}^{2}$ are linear maps such that for every edge $\sigma \subseteq \Delta^{3}$ the images $\square(\sigma)$ and $\mathrm{O}(\sigma)$ intersect, then there exists a partition $\alpha, \beta$ of $\Delta^{3}$ such that $\square(\alpha)$ and $\mathrm{O}(\beta)$ intersect. In Fig. 3 there are two such images of $\Delta^{3}$ (with vertices numbered by $\{1,2,3,4\}$ ), one whose vertices are labeled by squares, and one whose vertices are labeled by circles.

Observe that in this example all edges $\sigma \subseteq \Delta^{3}$ but $\{1,2\}$ are such that $\square(\sigma) \cap$ $\mathrm{O}(\sigma) \neq \varnothing$. However, there is no partition of $\{1,2,3,4\}$ into two parts $\alpha, \beta$ such that $\square(\alpha) \cap \mathrm{O}(\beta) \neq \varnothing$.

If we set $f_{1}=\cdots=f_{r}=f$ in Theorem 6 , then we obtain Tverberg's theorem. Therefore, the linearity of $f$ can be replaced by continuity according to the Topological Tverberg Theorem, which is proved only for prime numbers [3] and for powers of prime numbers [8].

It is easy to see that the topological version of Theorem 6 is false, even when the functions coincide on all vertices. Therefore, contrary to Tverberg's theorem, Theorem 6 can distinguish linear maps from continuous ones.

\section{Helly's Theorem}

Any point $p \in \mathbb{R}^{d}$ can be associated to the open semispace whose normal vector is $\overrightarrow{0 p}$. The following fact is well known (see [4]).

The origin is in the convex closure of a set of points in $\mathbb{R}^{d}$ if and only if the corresponding set of open semispaces is not intersecting.

Therefore the Very Colorful Carathéodory Theorem is equivalent to the following.

Proposition 7 (Very Colorful Helly Theorem for semispaces) Let $\mathcal{A}$ be a finite family of open semispaces in $\mathbb{R}^{d}$ colored with $d+1$ different colors. If every colorful subfamily $\mathcal{A}^{\prime}$ with $\left|\mathcal{A}^{\prime}\right|=d+1$ is intersecting, then there are two colors such that the subfamily of all sets of these colors is intersecting.

This proposition does not generalize to convex sets in $\mathbb{R}^{d}$. This can be seen from Fig. 4 where a yellow triangle, three red and three blue intervals fulfill the hypothesis, but only one color class is intersecting. 
Fig. 4 The sharpness of the Colorful Helly Theorem

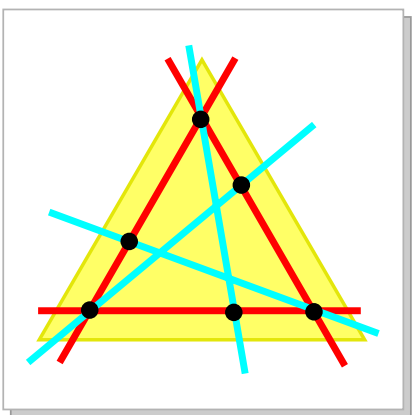

Later in this section we will come back to this phenomenon. The intersection of an open semispace with the unit sphere is an open hemisphere. Clearly, a set of open semispaces is intersecting if and only if the set of corresponding open hemispheres is intersecting, too. Therefore, Proposition 7 can be restated replacing semispaces by hemispheres.

Contrary to the affine case, this proposition does generalize to spherically convex sets. A spherically convex set is a subset $C$ of the sphere such that if $x, y \in C$, then $C$ contains all geodesics between $x$ and $y$. It is well known (and actually quite easy to check) that an open spherically convex set is the intersection of a collection of open hemispheres. A closed spherically convex set is the (topological) closure of an open spherically convex set or is the whole sphere.

Theorem 8 (Very Colorful Helly Theorem on the sphere) Let $\mathcal{A}$ be a finite family of closed, spherically convex sets in $\mathcal{S}^{d-1}$ colored with $d+1$ different colors. If every colorful subfamily $\mathcal{A}^{\prime}$ with $\left|\mathcal{A}^{\prime}\right|=d+1$ is intersecting, then there are two colors such that the subfamily of all sets of these colors is intersecting.

Proof Let $\mathcal{A}$ be a family contradicting the theorem. We can suppose that none of the elements of $\mathcal{A}$ is the whole sphere. For any colorful subfamily $\mathcal{A}^{\prime}$ with $\left|\mathcal{A}^{\prime}\right|=d+1$ we pick a point in the intersection $\bigcap_{A \in \mathcal{A}^{\prime}} A$, thus obtaining a finite set of points $P$. For each set $A \in \mathcal{A}$ denote by $A_{P}$ the set of all points in $P$ which we picked for the colorful subfamilies containing $A$. It is clear that $\left\{\operatorname{conv}\left\langle A_{P}\right\rangle \mid A \in \mathcal{A}\right\}$ is also a family contradicting the theorem. The convex sets in this new family are spherical polytopes, i.e., they are spanned by a finite set of vertices.

Suppose now that the elements of $\mathcal{A}$ are spherical polytopes. For each $A \in \mathcal{A}$ and $\varepsilon>0$ choose an open spherical polytope $A_{\varepsilon}$ which contains $A$ and is contained in the $\varepsilon$-neighborhood of $A$. It is clear that for a sufficiently small $\varepsilon$, the family $\mathcal{A}_{\varepsilon}=$ $\left\{A_{\varepsilon} \mid A \in \mathcal{A}\right\}$ also contradicts the theorem. Namely, there are no two colors such that the subfamily of all sets of these colors is intersecting. In other words, it is enough to prove the theorem for open spherical polytopes.

Now, let $\mathcal{A}$ be a family of open spherical polytopes contradicting the theorem. For every $A \in \mathcal{A}$ let $H_{A}$ be the finite set of open hemispheres such that $A$ is the intersection of them. We color every hemisphere in $H_{A}$ with the color of $A$.

By Proposition 7 there are two colors such that all hemispheres of these colors intersect. However, this implies that all $A \in \mathcal{A}$ of these two colors also intersect. 
We come back to the affine case now. As we saw before, we cannot obtain two intersecting colors. Lovász's Colorful Helly Theorem is sharp in this sense.

Theorem 9 (Colorful Helly Theorem [2]) Let $\mathcal{A}$ be a finite family of compact convex sets in $\mathbb{R}^{d}$ colored with $d+1$ different colors. If any colorful subfamily $\mathcal{A}^{\prime}$ with $\left|\mathcal{A}^{\prime}\right|=d+1$ is intersecting, then there is a color such that the subfamily of all sets of this color is intersecting.

We remark that using the presence of two colors in Theorem 8 one can give a new and simple proof of the Colorful Helly Theorem:

(1) Add a new compact convex set containing all sets in $\mathcal{A}$ and paint it with a new color.

(2) Lift the family to the sphere $\mathcal{S}^{d}$ in $\mathbb{R}^{d+1}$ using the projection from the center.

(3) Apply Theorem 8.

We shall further generalize the Colorful Helly Theorem in another direction. The following result is exactly the Colorful Helly Theorem in the case $m=d+1$.

Theorem 10 (Very Colorful Helly Theorem) Assume $m \in\{1, \ldots, d+1\}$. Let $\mathcal{A}$ be a finite family of compact convex sets in $\mathbb{R}^{d}$ colored with $d+1$ different colors. If every subfamily $\mathcal{A}^{\prime}$ with $\left|\mathcal{A}^{\prime}\right|=d+1$ and with at least $m$ different colors is intersecting, then there are $d+2-m$ colors such that the subfamily consisting of all sets of these colors is intersecting.

Proof A subfamily $\mathcal{A}^{\prime} \subseteq \mathcal{A}$ with $\left|\mathcal{A}^{\prime}\right|=d+1$ and with at least $m$ different colors is called rainbow. We denote $\widehat{\mathcal{B}}=\bigcap_{A \in \mathcal{B}} A$. The hypothesis of the theorem says that, for every rainbow subfamily $\mathcal{A}^{\prime}$, the set $\widehat{\mathcal{A}}^{\prime}$ is not empty.

Suppose the theorem is not true. Let $\mathbb{A}$ be the set of all families $\mathcal{A}$ contradicting the theorem such that $|\mathcal{A}|$ is minimal. For $\mathcal{A}$ and $\mathcal{B}$ in $\mathbb{A}$ we define the partial order relation $\mathcal{A} \preceq \mathcal{B}$ if there exists a bijection $\varphi: \mathcal{A} \rightarrow \mathcal{B}$ such that for all $A \in \mathcal{A}$ the inclusion $A \subseteq \varphi(A)$ holds.

Let $\mathcal{A}$ be a minimal element of this order relation and denote $\widetilde{\mathcal{A}}=\operatorname{conv} \bigcup_{A \in \mathcal{A}} A$. Let $p \in \widetilde{\mathcal{A}}$ be a point such that $p \notin \operatorname{conv}(\widetilde{\mathcal{A}} \backslash p)$, i.e., $p$ is an extreme point of $\widetilde{\mathcal{A}}$.

We claim that there exists a rainbow subfamily $\mathcal{B} \subseteq \mathcal{A}$ such that $\widehat{\mathcal{B}}=p$. Indeed, suppose this is not the case. Then for every rainbow subfamily $\mathcal{B}$ there exists a point $q(\mathcal{B}) \in \widehat{\mathcal{B}} \backslash p \subseteq \widetilde{\mathcal{A}} \backslash p$. For every $A \in \mathcal{A}$ denote by $A^{\prime}$ the convex closure of the points $q(\mathcal{B})$ such that $\mathcal{B}$ is a rainbow subfamily containing $A$. It is clear that the family $\left\{A^{\prime} \mid A \in \mathcal{A}\right\}=\mathcal{A}^{\prime} \preceq \mathcal{A}$ also contradicts the theorem. Moreover, $\mathcal{A}^{\prime} \neq \mathcal{A}$ because no set in $\mathcal{A}^{\prime}$ contains the point $p$. This contradicts the minimality of $\mathcal{A}$.

Now we prove that there exists a set $\mathcal{C} \subseteq \mathcal{B}$ with $|\mathcal{B} \backslash \mathcal{C}|=1$ such that $\widehat{\mathcal{C}}=p$. Indeed, suppose this is not the case. Then for every $\mathcal{C} \subseteq \mathcal{B}$ with $|\mathcal{C}|=d$ there exists a point $q(\mathcal{C}) \in \widehat{\mathcal{C}} \backslash p \subseteq \widetilde{\mathcal{A}} \backslash p$. Since $p$ is an extreme point, then there exists an affine hyperplane $H$ separating $p$ from the set of all $q(\mathcal{C})$. This means that for any $\mathcal{C} \subseteq \mathcal{B}$ with $|\mathcal{C}|=d$ we have that $\widehat{\mathcal{C}}$ intersects both affine open semispaces defined by $H$ and therefore $\widehat{\mathcal{C}}$ intersects $H$. Applying Helly's classical theorem in dimension $d-1$, we obtain that $\widehat{\mathcal{B}}$ intersects $H$. This contradicts the definition of $\mathcal{B}$. 
Let $\nabla$ be the set of colors of $\mathcal{C}$. We know that $|\nabla| \geq m-1$. If $|\nabla| \geq m$, then for any $A$ not in $\mathcal{C}$ the subfamily $\mathcal{C} \cup\{A\}$ is rainbow and intersecting. Therefore, all elements of $\mathcal{A}$ contain the point $p$. If $|\nabla|=m-1$, then for every $A \in \mathcal{A}$ whose color is not in $\nabla$ the subfamily $\mathcal{C} \cup\{A\}$ is rainbow and intersecting. Therefore, there are $d+1-|\nabla|=d+2-m$ colors such that all elements of $\mathcal{A}$ of these colors contain the point $p$. In both cases there is a contradiction.

Observe that Helly's theorem can be derived from this theorem in several ways, in particular, by setting $m=1$. Therefore, if one does not want to use Helly's theorem in the proof, then induction on the dimension can be applied.

\section{Hadwiger's Theorem}

The purpose of this last section is to prove the following.

Theorem 11 (Very Colorful Hadwiger Theorem) Let $A_{1}, \ldots, A_{n}$ be a finite, ordered family of compact convex sets in the plane colored with at least $m \geq 3$ different colors. If for any choice of differently colored $A_{i}, A_{j}$ and $A_{k}$ with $i<j<k$ the condition $A_{j} \cap\left\langle A_{i} \cup A_{k}\right\rangle \neq \varnothing$ holds, then there are $m-2$ colors such that there is a line transversal to all the convex sets of these colors.

This theorem was proved in [1] for the particular case $m=3$. It is not easy to prove Theorem 11 from this particular case. However, the proof given in [1] can be modified to prove Theorem 11. Since the needed modifications are technical, it makes no sense to repeat the paper [1]. Therefore, the proof given here is not self-contained. We will only modify the definitions of the following concepts from [1]:

- middle line of a colored family

- middle colored separating sign vector

- balanced colored sign vector

and we will prove Lemmas 3 and 4 from [1] in this more general context. This will be enough to conclude the proof of Theorem 11.

Before proceeding, we remark that Theorem 11 is sharp in the sense that one cannot find a transversal line to the sets of more than $m-2$ colors. This can be seen from the example in Fig. 5.

Now we start to discuss the promised modifications of [1]. Let $d$ be a direction in the plane and $d^{\perp}$ its orthogonal direction. Chose any oriented line $\ell^{\perp}$ in the direction of $d^{\perp}$. When we orthogonally project any convex set $A$ to the line $\ell^{\perp}$ we obtain an interval $[i(A), j(A)]$ and we can think that $i(A)$ and $j(A)$ are real numbers. Let $A_{1}, \ldots, A_{n}$ be a colored family of plane convex sets. For any color $c$ define $q_{c}=$ $\operatorname{Sup}\left\{i\left(A_{i}\right) \mid A_{i}\right.$ is colored $\left.c\right\}$ and $p_{c}=\operatorname{Inf}\left\{j\left(A_{i}\right) \mid A_{i}\right.$ is colored $\left.c\right\}$. Moreover, if $K$ is any subset of colors, then we define $q_{K}=\operatorname{Sup}\left\{q_{c} \mid c \in K\right\}$ and $p_{K}=\operatorname{Inf}\left\{p_{c} \mid c \in K\right\}$. It is easy to see that there is a line transversal in the direction $d$ to all the sets colored with the colors in $K$ if and only if $q_{K} \leq p_{K}$.

Denote by $C$ the set of all colors and suppose $|C|=m \geq 3$. Then by $u_{1}, u_{2}, \ldots, u_{m}$ we can denote real numbers such that $u_{1} \leq u_{2} \leq \cdots \leq u_{m}$ and $\left\{u_{1}, u_{2}, \ldots, u_{m}\right\}=$ 
Fig. 5 The sharpness of the Very Colorful Hadwiger Theorem

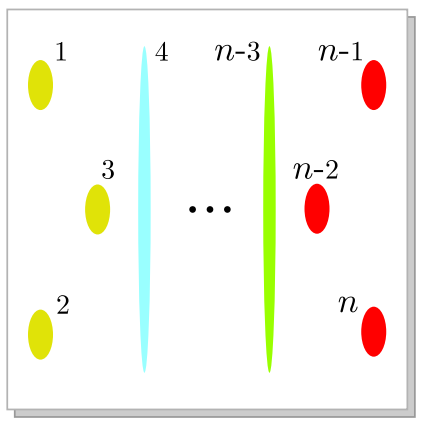

$\left\{p_{c} \mid c \in C\right\}$. In the same way we define $v_{1}, v_{2}, \ldots, v_{m}$ such that $v_{1} \geq v_{2} \geq \cdots \geq v_{m}$ and $\left\{v_{1}, v_{2}, \ldots, v_{m}\right\}=\left\{q_{c} \mid c \in C\right\}$.

Let $\ell$ be the oriented line in the direction $d$ which meets $\ell^{\perp}$ in the point $\theta=$ $\left(u_{2}+v_{2}\right) / 2$. We will call $\ell$ the middle line of the colored family $\mathcal{A}$ in the direction $d$. The colored separating sign vector of the line $\ell$ will be called the middle colored separating sign vector of the direction $d$.

A colored sign vector will be called balanced if the following conditions hold:

(1) The number of different colors of non-zero coordinates is at least three.

(2) The number of different colors of positive coordinates is at least two.

(3) The number of different colors of negative coordinates is at least two.

Lemma 12 ([1] Lemma 3) If the $m$-colored family $\mathcal{A}$ has the property that for any subset of colors $K$ with $|K|=m-2$ the subfamily of all sets colored with the colors in $K$ has no transversal line in the direction $d$, then the middle colored separating sign vector of $\mathcal{A}$ in the direction $d$ is balanced.

Proof We shall see that $u_{2}<v_{2}$. If not, then $u_{m} \geq \cdots \geq u_{2} \geq v_{2} \geq \cdots \geq v_{m}$. Let $P$ be the set of colors such that $\left\{u_{2}, \ldots, u_{m}\right\}=\left\{p_{c} \mid c \in P\right\}$, and $Q$ be the set of colors such that $\left\{u_{2}, \ldots, u_{m}\right\}=\left\{q_{c} \mid c \in Q\right\}$. Let $K=P \cap Q$. We have $|K| \geq m-2$ and $p_{K} \geq p_{P} \geq q_{Q} \geq q_{K}$. This implies that there is a line transversal in the direction $d$ to all sets colored with the colors in $K$ contradicting the hypothesis.

So $u_{1} \leq u_{2}<\theta<v_{2} \leq v_{1}$, which means that there are positive coordinates of two different colors (those corresponding to $v_{1}$ and $v_{2}$ ) and there are negative coordinates of two different colors. If the number of different colors of non-zero coordinates is exactly two, then the line $\ell$ would be transversal to all the sets colored with the other $m-2$ colors.

Lemma 13 ([1] Lemma 4) If $\mathbf{x} \preceq \mathbf{y}$ are both balanced and Hadwiger colored sign vectors, then they have the same sign.

Proof Assume that $\mathbf{x}$ and $\mathbf{y}$ contradict the lemma. Say that the sign of $\mathbf{x}$ is plus and the sign of $\mathbf{y}$ is minus. Denote by $a$ and $b$ the indices of the leading coordinates of $\mathbf{x}$ and $\mathbf{y}$, respectively. As is shown in [1] we can suppose that $b=1$ and $a=2$.

Since $\mathbf{x} \preceq \mathbf{y}$, then $x_{2}=y_{2}=+$. We also know that $x_{1}=0$ and $y_{1}=-$. We divide the proof into two cases: the colors of coordinates 1 and 2 coincide or not. 
Suppose they coincide. Since $\mathbf{y}$ is balanced, then there must be a coordinate (say $i$ ) of a second color such that $y_{i}=-$ and a coordinate (say $j$ ) of a third color such that $y_{j}=+$. If $i<j$, then $\left(y_{2}, y_{i}, y_{j}\right)=(+-+)$, otherwise $\left(y_{1}, y_{j}, y_{i}\right)=(-+-)$, and both cases are contradictory to the fact that $\mathbf{y}$ is Hadwiger.

Suppose the first coordinate is red and the second is blue. If there exists a coordinate (say $i$ ) of another color such that $x_{i}=y_{i}=-$, then $\left(y_{1}, y_{2}, y_{i}\right)=(-+-)$, and this is contradictory to the fact that $\mathbf{y}$ is Hadwiger. So, every color different from red and blue has positive coordinates.

Since $\mathbf{x}$ is balanced and $\mathbf{x} \preceq \mathbf{y}$, then there must exist:

A coordinate (say $i$ ) of a third color such that $x_{i}=y_{i}=+$.

A red coordinate (say $j$ ) such that $x_{j}=y_{j}=-$.

A blue coordinate (say $k$ ) such that $x_{k}=y_{k}=-$.

If $j<i$, then $\left(y_{2}, y_{j}, y_{i}\right)=(+-+)$. Hence, $i<j$. If $k<i$, then $\left(y_{k}, y_{i}, y_{j}\right)=$ $(-+-)$; otherwise $\left(y_{1}, y_{i}, y_{k}\right)=(-+-)$ and in all cases we obtain a contradiction.

This concludes the required modifications of the proof in [1].

Remark During the preparation of this paper, János Pach informed us that he, Andreas Holmsen and Helge Tverberg simultaneously and independently discovered Theorems 1 and 8 .

\section{References}

1. Arocha, J.L., Bracho, J., Montejano, L.: A colorful theorem on transversal lines to plane convex sets. Combinatorica 28(4), 379-384 (2008)

2. Bárány, I.: A generalization of Carathéodory's theorem. Discrete Math. 40(2-3), 141-152 (1982)

3. Bárány, I., Shlosman, S.B., Szúcs, A.: On a topological generalization of a theorem of Tverberg. J. Lond. Math. Soc. 23(1), 158-164 (1981)

4. Danzer, L., Grünbaum, B., Klee, V.: Helly's theorem and its relatives. In: Proc. Sympos. Pure Math., vol. VII, pp. 101-180. Am. Math. Soc., Providence (1963)

5. Montejano, L., Oliveros, D.: Colourful transversal theorems. Contributions Discrete Math. 3(2), 60-75 (2008)

6. Pór, A.: Diploma Thesis, Eötvös University, Budapest (1998)

7. Sarkaria, K.S.: Tverberg's theorem via number fields. Isr. J. Math. 79(2-3), 317-320 (1992)

8. Volovikov, A.Yu.: On a topological generalization of the Tverberg theorem. Math. Notes 59(3), 324326 (1996) 